\title{
LINEAR PARAMETRICALLY VARYING SYSTEMS WITH BRIEF INSTABILITIES: AN APPLICATION TO INTEGRATED VISION / IMU NAVIGATION ${ }^{\star}$
}

\author{
João Hespanha' ${ }^{1}$ Oleg Yakimenko ${ }^{2}$, Isaac Kaminer ${ }^{3}$, and António Pascoal ${ }^{4}$
}

\begin{abstract}
.
This paper studies linear parametrically varying systems (LPVs) with brief instabilities. LPVs are ubiquitous because they provide an elegant, albeit conservative framework for the study of nonlinear systems. This is done by analyzing a related family of linear timeinvariant systems parameterized by a parameter $p$ that lives in some compact set. In the conventional set-up of LPV theory, it is usually required that the system matrices in the family of parameterized linear systems be stable for all values of $p$. However, there are interesting problems for which this requirement does not hold true, that is, the linear system matrices are unstable for some of values of the parameter $p$, instability occurring for brief instants of time only. This paper introduces the concept of LPVs with brief instabilities and derives tools for stability and performance analysis of these systems, where performance is evaluated in terms of $L_{2}$ induced norms. The main results show that stability and performance can be assessed by examining the feasibility of parameterized sets of Linear Matrix Inequalities (LMIs). An application to the problem of designing a nonlinear vision/inertial navigation filter for an aincraft approaching an aircraft carrier is included. The results developed provide the proper framework to deal with out-of-frame events that arise when the vision system loses its target temporarily. Field tests with a prototype unmanned air vehicle illustrate the performance of the filter and illustrate the scope of applications of the new theory developed.
\end{abstract}

1 Introduction

It is often possible to express the dynamics of a nonlinear system as

$$
\dot{x}=A(\rho(x)) x, x \in \mathfrak{R}^{n},
$$

where the function $\rho$ takes values in some "parameter" set $P$ and $\{A(\rho): \rho \in P\}$ can be viewed as a family of matrices parameterized by the elements of the set $P$. This motivates the study of linear parametrically varying systems (LPVs) that are simply defined as

$$
\dot{x}=A(p(t)) x, x \in \mathfrak{R}^{n},
$$

where $p$ is an arbitrary signal taking values in the parameter set $P$. Since every solution to the nonlinear system (1) is a solution to the linear time-varying system (2) (for an appropriately defined signal $p(t)$ ), LPVs allow one to prove stability-like properties of a nonlinear system by analyzing a family of time-varying linear systems. The price paid for this simplification is the conservativeness that arises from the fact that the set of solutions to (2) is generally much larger than the set of solutions to (1). This paper attempts to reduce the conservativeness of this type of design by considering restricted classes of signals $p$.

If one assumes that any piecewise-continuous signal $p$ is al-

\footnotetext{
- This work was supported by the Office of Naval Research under contract No. N0001497AF00002.

'Department of Electrical Engineering, University of Southern California, Los Angeles CA 90089, USA.

2 Department of Acronautical and Astronautical Engineering, Naval Postgraduate School, Monterey, CA 93943, USA.

${ }^{3}$ Department of Aeronautical and Astronautical Engineering, Naval Postgraduate School, Monterey, CA 93943, USA.

${ }_{4}^{4}$ Department of Electrical Engineering and Institute for Systems and Robotics, Torre Norte, Instituto Superior Técnico, Av. Rovisco Pais, 1, 1049-001, Lisbon, Portugal.
}

required to guarantee boundedness of any solution to (2). However, this is no longer the case when $A(p)$ is temporarily unstable. This paper shows how, with an appropriate notion of "brief instability", it is possible that an LPV remain uniformly exponentially stable even when some of the matrices $A(p)$ are unstable for brief periods of time. Necessary conditions for this to occur are cast in terms of a parameterized family of Linear Matrix Inequalities (LMIs). Furthermore, the paper analyzes the impact of brief instabilities on the performance of an LPV system, as measured in terms of its $L_{2-}$ induced norm. In particular, a parameterized set of LMIs is derived that, when feasible, provides an upper bound on the $L_{2}$-induced norm of an LPV system with brief instabilities.

Often, it is not possible to satisfy the parameterized LMIs for all values of the parameter $p$. To deal with this situation, "local" versions of the results above are provided in which it is assumed that the state of (1) starts inside an ellipsoid, thus restricting the values that

$$
p(t)=\rho(x(t)), t \geq 0
$$

can take. These results explore directly the fact that the LPV system (2) is an abstraction of the more complex nonlinear system (1).

The analysis of LPV systems with brief instabilities is inspired by previous work of the first author on switched systems [22, 23], as well as by the work reported in $[24,25]$. Switched systems can be viewed as a form of LPV systems where the signal $p(t)$ in (2) is restricted to be constant between two consecutive discontinuities. The idea of brief instabilities was introduced in [24] for switched systems, where the authors provide conditions for exponential stability of switched system with brief instabilities. These results were extended in [25] for $L_{2}$ disturbance attenuation.

The work reported in this paper is also closely related to that described in [26], where the authors provide conditions for the stability of Asynchronous Dynamical Systems (ADSs). The latter that can also be viewed as a particular form of switched systems for which the system dynamics change in response to extemal asynchronous events. These events may make the system become unstable for certain periods of time. In [26] the authors provide LMIs that guarantee exponential convergence of the state of ADS. Feasibility of the LMIs requires that the periods of instability occur for a small fraction of the time. Because the authors of [26] only consider asymptotic rates for the occurrence of the events that trigger changes in the dynamics, their results are only asymptotic and do not provide uniform bounds on the state.

In this paper, the results on LPV systems with brief instabilities are shown to provide a new framework for the design of navigation filters that rely on vision and inertial sensors. See [10] for an introduction to this problem and its application to the design of a navigation system for an aircraft approaching an aircraft carrier under the constraint that only passive sensors be used. The basic nonlinear filter structure adopted is described in [10], where the authors have derived sufficient conditions for the existence of nonlinear integrated vision/inertial filters with guaranteed regional stability and perform-

\footnotetext{
${ }^{5}$ Although in [24] the authors consider a slightly more conservative definition of brief instabilities, their results seem to be easily extendable to the definition given in Section 2.
} 
ance. However, they did not address the fact that instabilities do occur when the vision system that is used to compensate for the drift that is introduced by inertial sensors cannot be used temporarily because of out-of-frame events, i.e., periods of time when the vision system is unable to see the target due to occlusions or the limited field of view. The results in [10] are extended in this paper to accommodate out-offrame events.

The paper is organized as follows. In section 2 the stability and performance results on the LPV systems with brief instabilities are introduced. Section 3 applies the theory developed in Section 2 to the design of an integrated vision/inertial filter. This section also includes description of the experimental setup used to test the filter performance. The paper ends with conclusions.

\section{LPVs with Brief Instabilities}

Consider the homogeneous Linear Parameter Varying (LPV) system

$$
\Sigma_{p}:=\dot{x}=A(p) x, y=C(p) x,
$$

where $p$ denotes a piecewise-continuous' time-varying parameter taking values in the set $P \subset \mathfrak{R}^{k}$ and $A: P \rightarrow \mathfrak{R}^{n \times n}$ and $C$ : $P \rightarrow \Re^{m \times m}$ are functions that map the parameter set to the system dynamics. In what follows $P_{\text {stable }}$ denoted the subset of $P$ for which $A(p)$ is a stability matrix, that is, $A(p)$ is stable if and only if $p \in P_{\text {stable }}$. The remaining elements of $P$ form the set $P_{\text {unstable }}$. We assume that $P$ is a compact subset of a finite dimensional space and that $A$ and $C$ are continuous functions. Because of these assumptions, it is straightforward to show that $P_{\text {unstable }}$ is also compact. In the sequel we derive conditions on $p$ that are sufficient to guarantee that $x$ converge to zero exponentially fast. We will also compute an upper bound on the transient response of the output $y$.

For a given time-varying parameter $p$ and $t>\tau>0$, let $T_{p}(\tau, t)$ denote the amount of time in the interval $(\tau, t)$ that $p$ remains in $P_{\text {unstable }}$. Formally,

$$
T_{p}(\tau, t):=\int \chi(p(s)) d x,
$$

where $\chi: P \rightarrow\{0,1\}$ denotes the characteristic function of $P_{u n s t a b l e}$, i.e.,

$$
\chi(p):= \begin{cases}0 & p \in P_{\text {stable }} \\ 1 & p \in P_{\text {unstable }}\end{cases}
$$

The integral in (4) is well defined because the piecewisecontinuity of $p$ and the compactness of $P_{\text {unstable }}$ guarantee that $\chi(p)$ is also piecewise-continuous. We will say that $\Sigma_{p}$ has brief instabilities if

$$
T_{p}(\tau, t) \leq T_{0}+\alpha(t-\tau), \forall t \geq \tau \geq 0,
$$

for some $T_{0} \geq 0, \alpha \in[0.1]$. The scalar $T_{0}$ is called the instability bound and $\alpha$ the asymptotic instability ratio.

\subsection{Stability}

We now provide conditions under which system (3) is stable in the presence of brief instabilities.

Lemma 2.1 Consider the LPV system $\Sigma_{p}$ defined by (3) and assume there exist positive definite matrices $R \in \mathfrak{R}^{m \times m}$ and $X \in \mathfrak{R}^{n \times n}$ and positive scalars $\lambda_{0}$, $\mu$ such that

\footnotetext{
${ }^{1}$ We say that a signal $v:[0, \infty) \rightarrow \mathfrak{R}^{k}$ is piecewise continuous if $v$ has a finite number of discontinuities on any finite interval.
}

$$
\begin{gathered}
A(p)^{T} X+X A(p) \leq-\lambda_{0} X, \forall p \in P_{\text {stable }}, \\
A(p)^{T} X+X A(p) \leq \mu P, \forall p \in P_{\text {unstuble }},
\end{gathered}
$$

and

$$
X \geq C(p)^{T} R C(p), \forall p \in P .
$$

Further assume that $\Sigma_{p}$ has brief instabilities with instability bound $T_{0}$ and asymptotic instability ratio $\alpha<\alpha^{*}=\frac{\lambda_{0}}{\lambda_{0}+\mu}$.

Then, $x$ and $y$ converge to zero exponentially and $y(t)^{T} R y(t) \leq e^{\left(\lambda_{0} \mu\right) T_{0}} x(0)^{T} X x(0) ; \forall t \geq 0$, along solutions of (3).

Note. When (5) holds, (6) will always hold for sufficiently large . Moreover, we can always scale $P$ so that (7) also holds.

Proof: For a particular solution $x$ of (3), let

$$
V(t):=x(t)^{T} X x(t) \text {. }
$$

From (5)-(6) it follows that $\mathscr{W}^{\forall} \leq-\lambda_{0} V$ while $p \in P_{\text {stable }}$ and $\not \forall \leq \mu V$ while $p \in P_{\text {unstable. }}$. Therefore,

$$
V(t) \leq e^{-\lambda_{0}\left(t--T_{p}(, t)\right)+\mu T_{p}(, t)} V(\tau), \forall t \geq \tau \geq 0 .
$$

By assumption, $\Sigma_{p}$ has brief instabilities with instability bound $T_{0}$ and asymptotic instability ratio . Let $\lambda:=\lambda_{0}-\alpha\left(\lambda_{0}+\mu\right)$. Then,

$$
\begin{gathered}
-\lambda_{0}\left(t-\tau-T_{p}(\tau, t)\right)+\mu T_{p}(\tau, t) \leq\left(\lambda_{0}+\mu\right) T_{0}-\lambda(t-\tau),(9) \\
\forall t \geq \tau \geq 0 . \text { Using }(8) \text { and }(9) \text { yields } \\
V(t) \leq e^{\left(\lambda_{0} \mu T_{0}-\lambda(t-\tau)\right.} V(\tau), \forall t \geq \tau \geq 0 .
\end{gathered}
$$

Furthermore, (7) implies that

$$
y(t)^{T} R y(t) \leq x(t)^{T} X x(t) \leq e^{\left(\lambda_{0} \mu\right) T_{0}-\lambda t} x(0)^{T} X x(0)
$$

for every $t \geq 0$, thus completing the proof.

The results above shows that $x^{T} X x$ and $y^{T} R y$ decay exponentially along solutions of (3) provided that $\lambda>0$.

LPV models such as (3) are often used to model nonlinear systems where the time-varying parameter $p$ is a function of the state, e.g.,

$$
p(t):=f(x(t), t),
$$

where $f: \Re^{n} \times[0, \infty) \rightarrow P$. When this happens (5)-(7) often do not hold globally and a local version of Lemma 2.1 is needed. Take a positive definite matrix $R \in \mathfrak{R}^{m \times m}$ and consider the set of states for which the output $y$ is guaranteed to be in the ellipsoid defined by $y^{T} R y \leq 1$, i.e.,

$$
\Omega:=\left\{z \in \mathfrak{R}^{n}: z^{T} C(p)^{T} R C(p) z \leq 1, \forall p \in P\right\} .
$$

We now consider a version of Lemma 2.1 that is local to the set $\Omega$. To this effect, suppose that there exists a symmetric positive definite matrix $P \in \mathfrak{R}^{n \times n}$ and positive scalars $\lambda_{0}$, for which

$$
\begin{gathered}
A(p)^{T} X+X A(p) \leq-\lambda_{0} X, \forall t: x(t) \in \Omega, p(t) \in P_{\text {stable }}, \\
A(p)^{T} X+X A(p) \leq \mu X, \forall t: x(t) \in \Omega, p(t) \in P_{\text {unstable }}, \\
X \geq C(p)^{T} R C(p), \forall t: x(t) \in \Omega .
\end{gathered}
$$

By requiring that the initialization of (3) satisfy $e^{\left(\lambda_{0} \mu\right) T_{0}} x(0)^{T} X x(0)<1$, it is straightforward to prove by contradiction (cf. equation (10)) that $x(t)$ will always remain inside $\Omega$ along solutions to (3). The following corollary of Lemma 2.1 is thus proved. 
Corollary 2.2 Assume that (11)-(13) hold. Suppose that $\Sigma_{p}$ has brief instabilities with instability bound $T_{0}$ and asymptotic instability $\alpha<\alpha^{*}$, and assume that $x(0)^{T} X x(0) \leq e^{-\left(\lambda_{0}+\mu\right) T_{0}}$. Then, $x$ converges to zero exponentially fast along solutions of (3), without leaving $\Omega$.

\subsection{Performance}

Suppose now that an input and an extra output are added to the LPV system considered in Section 2.1 to obtain the new system

$\Sigma_{p}:=\dot{x}=A(p) x+B(p) u, y=C(p) x, z=D(p) x$.

The analysis that follows shows how to compute the $L_{2}$-induced norm from $u$ to $z$ when $p$ has brief instabilities.

Lemma 2.3 Consider the LPV system $\underline{\Sigma}_{p}$ defined by (14) and assume there exist positive definite matrices $R \in \mathfrak{R}^{m \times m}$ and $X \in \mathfrak{R}^{n \times n}$ and positive scalars $\lambda_{0}, \mu$ and $\gamma$ such that

$$
\begin{array}{ll}
\mathfrak{I} \leq-\lambda_{0} X, & p \in P_{\text {stable }}, \\
\mathfrak{I} \leq \mu X, & p \in P_{\text {unstable }},
\end{array}
$$

and

$$
X \geq C(p)^{T} R C(p), \quad \cdot p \in P .
$$

where

$$
\mathfrak{J}=A(p)^{T} X+X A(p)+X B(p) B(p)^{T} X+\frac{D(p) D(p)^{T}}{\gamma^{2}}
$$

Suppose $\Sigma_{p}$ has brief instabilities with instability bound $T_{0}$ and asymptotic instability ratio $\alpha<\alpha^{*}$ and that $u$ is bounded. Then $x$ and $y$ remain bounded along solutions of (14), with

$$
y(t)^{T} R y(t) \leq e^{\left(\lambda_{0}+\mu\right) T_{0}}\left(x(0)^{T} X x(0)+\int\|u(s)\|^{2} d s\right), \forall t \geq 0 .
$$

Moreover, the $L_{2}$-induced norm from $u$ to $z$ is no larger than $\gamma \sqrt{\frac{e^{\left(\lambda_{0}+\mu\right) T_{0}} \lambda_{0}}{\lambda_{0}-\alpha\left(\lambda_{0}+\mu\right)}}$ and both $x$ and $y$ converge to zero if $u \in L_{2}$.

Proof: For a particular solution $x$ of (14), let $V(t):=x(t)^{T} X x(t)$. Consider now an interval $\left(t_{1}, t_{2}\right)$ on which $p \in P_{\text {stable }}$. From (15) it follows that

on this interval and therefore

$$
V \leq-\lambda_{0} V+\|u\|^{2}-\|z\|^{2}-2
$$

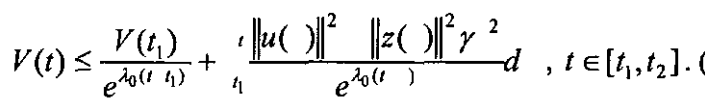

Similarly, on an interval $\left(t_{2}, t_{3}\right)$ on which $p \in P_{\text {unstable }}$, it follows from (16) that

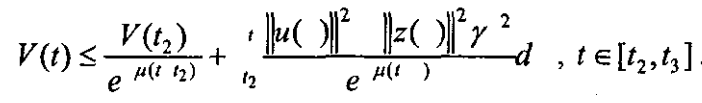

Iterating (18) and (19) over consecutive intervals yields

$$
V(t) \leq \frac{V(\tau)}{e^{\lambda_{0}(t} \frac{\left.r_{p}(t,)\right) \mu T_{p}(, t)}{2}}+\frac{\|u(s)\|^{2}\|z(s)\|^{2}{ }^{2}}{e^{\lambda_{0}\left(t s T_{p}(s, t)\right) \mu T_{p}(s, t)}} d s
$$

$\forall t \geq \tau \geq 0$. Using the above relationship, the two following inequalities are also obtained for $\forall t \geq \tau \geq 0$ :

$$
V(t) \leq \frac{V(\tau)}{\left.e^{\lambda_{0}(t} T_{p}(, t)\right) \mu T_{p}(, t)}+{ }^{t} \frac{\|u(s)\|^{2}}{e^{\lambda_{0}\left(t s T_{p}(s, t)\right) \mu T_{p}(s, t)}} d s
$$

$$
\begin{aligned}
\frac{\|z(s)\|^{2}}{\gamma^{2} e^{\lambda_{0}\left(t s T_{p}(s, t)\right) \mu T_{p}(s, t)} d s \leq} \leq \frac{V()}{e^{\lambda_{0}\left(t \quad T_{p}(, t) \mu T_{p}(, t)\right.}} \\
\\
{ }^{\prime} \frac{\|u(s)\|^{2}}{e^{\lambda_{0}\left(t s T_{p}(s, t)\right) \mu T_{p}(s, t)}} d s .
\end{aligned}
$$

Suppose now that $\ldots$ p has brief instabilities with instability bound $T_{0}$ and asymptotic instability ratio , that is, (9) holds with $\lambda:=\lambda_{0}-\alpha\left(\lambda_{0} \quad \mu\right)$. From (9) and (20) it can be concluded that

$$
V(t) \leq e^{\left(\lambda_{0}+\mu\right) T_{0} \lambda\left({ }^{t}\right)} V() \quad \frac{\|u(s)\|^{2}}{e^{\left(\lambda_{0}+\mu\right) T_{0}+\lambda(t s)}} d s ; \forall t \geq \tau \geq 0 .
$$

Using (7), this can be shown to imply

$$
\begin{aligned}
y(t)^{T} R y(t) & \leq x(t)^{T} X x(t) \\
& \leq \frac{x(0)^{T} X x(0)}{e^{\left(0^{0}+\mu\right) T_{0}+t}} \quad e^{\left({ }^{0}+\mu\right) T_{0}} \frac{t\|u(s)\|^{2}}{e^{(t s)}} d s .
\end{aligned}
$$

Using (9) in (21) and the fact that $\left(\lambda_{0} \quad \mu\right) T_{p}(s, t) \geq 0$ yields

$$
\frac{\|z(s)\|^{2}}{\gamma^{2} e^{\lambda_{0}(t s)}} d s \leq \frac{V()}{e^{\left(\lambda_{0}+\mu\right) T_{0}+\lambda(t)}}+{ }^{\prime} \frac{\|u(s)\|^{2}}{e^{\left(\lambda_{0}+\mu\right) T_{0}+\lambda(t s)}} d s,
$$

gives

$$
\frac{1}{\gamma^{2}}{ }^{\infty} \cdot \frac{\|z(s)\|^{2}}{e^{\lambda_{0}(t s)}} d s d t \leq \frac{V()}{e^{\left(\lambda_{0}+\mu\right) T_{0}}}+\infty \frac{\|u(s)\|^{2}}{e^{\left(\lambda_{0}+\mu\right) T_{0}+\lambda(t s)}} d s .
$$

Exchanging the order of integration, it is easy to show that

$$
\frac{1}{\lambda_{0} \gamma^{2}}\|z(s)\|^{2} d s \leq \frac{e^{\left(\lambda_{0}+\mu\right) T_{0}}}{\lambda}\left(V()+\|u(s)\|^{2} d s\right)
$$

thus completing the proof.

A local version of Lemma 2.3 is derived next. To this effect take a positive definite matrix $R \in \mathfrak{R}^{m \times m}$ and consider the set of states for which the output $y$ is guaranteed to be in the ellipsoid defined by $y^{T} R y<1$, i.e.,

$$
\Omega:=\left\{z \in \mathfrak{R}^{n}: z^{T} C(p)^{T} R C(p) z \leq 1, \forall p \in P\right\} .
$$

Suppose now that there exists a symmetric positive definite matrix $P \in \mathfrak{R}^{n \times n}$ and positive scalars $\lambda_{0}$, and $\gamma$ such that

$$
\begin{aligned}
& \mathfrak{I} \leq-\lambda_{0} X, \quad \forall t: x(t) \in \Omega, p(t) \in P_{\text {stable }}, \\
& \mathfrak{I} \leq \mu X, \forall t: x(t) \in \Omega, p(t) \in P_{\text {unstable }}, \\
& X \quad C(p)^{T} R C(p), \forall t: x(t) \in \Omega,
\end{aligned}
$$

where $\mathfrak{I}$ is defined as (17). The following corollary of Lemma 2.3 is then straightforward to derive.

Corollary 2.4 Assume that (23)-(25) hold. Suppose that ${ }_{-}$has brief instabilities with instability bound $T_{0}$ and asymptotic insta-

bility ratio $\alpha<\alpha^{*}$, that $u$ is bounded, and that $x(0)^{T} X x(0)+\int_{0} \mid u(s) \|^{2} d s \leq e^{\left(\lambda_{0}+\mu\right) T_{0}}$. Then, $x$ converges to zero along solutions of (3) without leaving the set $\Omega$ Furthermore, the $L_{2}$-induced norm from $u$ to $z$ is no larger than $\gamma \sqrt{\frac{e^{\left(\lambda_{0}+\mu\right) T_{0}} \lambda_{0}}{\lambda}}$. and 
3 Application: Design of integrated vision/inertial filters In this section we apply the ideas formulated above to the design of integrated vision/inertial filters. A basic filtering structure has been introduced in [10], where the authors obtained sufficient conditions for the existence of nonlinear integrated vision/inertial filters with guaranteed regional stability and performance. These results are extended in this paper to include so-called out-of-frame events.

\subsection{Problem formulation}

This section introduces the navigation problem that is the main focus of the paper and describes its mathematical formulation in terms of an equivalent filter design problem. For the sake of clarity, we first introduce some required notation and review the kinematic relationships of an aircraft / ship carrier ensemble, where the former is equipped with a vision based system.

Consider Figure 1, which depicts an aircraft equipped with a vision camera operating in the vicinity of a ship. Let $\{I\}$ denote an inertial reference $\{B\}$ a body-fixed frame that moves with the aircraft and $\{C\}$ a camera-fixed frame. The symbol $\{S\}$ denotes a ship-fixed body frame. The following symbols will be used (soe Figure 1):

- $\mathbf{p}_{B}=\left[\begin{array}{lll}x_{B} & y_{B} & z_{B}\end{array}\right]^{T}$ - position of the origin of $\{B\}$ measured in \{l) (i.e., inertial position of the aircraft);

- $\mathbf{p}_{S}=\left[\begin{array}{lll}x_{s} & y_{S} & z_{S}\end{array}\right]^{T}$ - inertial position of the ship;

- $\mathbf{p}_{S B}\left(a b b v . \mathbf{p}=\left[\begin{array}{ll}x & y z\end{array}\right]^{T}\right)$ - relative position of the ship with respect to the aircraft, resolved in $\{I\}$;

- ${ }^{B} \mathbf{p}_{S B}\left(a b b v . \mathbf{p}_{c}=\left[\begin{array}{lll}x_{c} & y_{c} z_{c}\end{array}\right]^{T}\right)$-relative position of the ship with respect to the aircraft, resolved in $\{C\}$ :

- $\quad \mathbf{v}_{B}$ - linear velocity of the origin of $\{B\}$ measured in $\{I\}$ (i.e., inertial velocity of the aircraft);

- $\mathbf{v}_{S}$ - inertial velocity of the ship;

- ${ }^{B} \mathbf{a}$ - linear acceleration of $\{B\}$ with respect to $\{l\}$, resolved in $\{B\}$;

- $\quad \omega$ - angular velocity of $\{C\}$ with respect to $\{I\}$, resolved in $\{I\}$ $\left(\omega=\left[\omega_{x} \omega_{y} \omega_{z}\right]^{T}\right)$;

- $\Lambda=[\phi \theta \psi]^{T}$ - vector of roll, pitch, and yaw angles that parameterize locally the orientation of frame $\{C\}$ with respect to $\{1\}$.

Given two frames $\{A\}$ and $\{B\},{ }_{B}^{A} R$ denotes the rotation matrix from $\{B\}$ to $\{A\}$. In particular, ${ }_{C}^{I} R$ (abbreviated $R$ ) is the rotation matrix from $\{C\}$ to $\{I\}$, parameterized locally by $\Lambda$, that is, $R=R(\Lambda)$

\subsection{Kinematic relations}

The rotation matrix $R$ satisfies the orthonormality condition $R^{T} R=1$. Furthermore, [12]:

$$
\dot{R}=R S(\boldsymbol{\omega}),
$$

where

$$
S(\omega):=\left[\begin{array}{ccc}
0 & -\omega_{z} & \omega_{y} \\
\omega_{z} & 0 & -\omega_{x} \\
-\omega_{y} & \omega_{x} & 0
\end{array}\right],
$$

is a skew symmetric matrix, that is, $S^{T}=-S$. The matrix $S$ satisfies the relationship $S(\mathbf{a}) \mathbf{b}=\mathbf{a} \times \mathbf{b}$, where $\mathbf{a}, \mathbf{b}$ are arbitrary vectors and $\times$ denotes the cross product operation. Furthermore, $\|S(\omega)\|=\|\omega\|$.

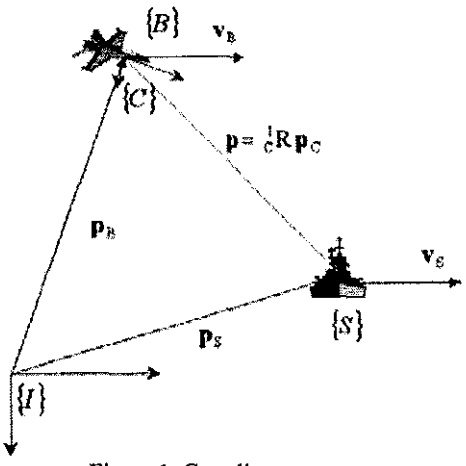

Figure 1: Coordinate systems

We introduce the following assumption:

A1 - the ship 's inertial velocity $\mathbf{v}_{S}$ is constant and different from zero.

From the above definitions, it follows that

$$
\mathbf{p}_{S}=\mathbf{p}_{B}-{ }_{C}^{I} R \mathbf{p}_{C} \Rightarrow \frac{d^{2}}{d t^{2}}\left({ }_{C}^{I} R \mathbf{p}_{C}\right)=\frac{d^{2}}{d t^{2}} \mathbf{p}_{S}-\frac{d^{2}}{d t^{2}} \mathbf{p}_{B},
$$

and since $\frac{d^{2}}{d t^{2}} \mathbf{p}_{s}=0$ (assumption $\mathbf{A} 1$ ) we obtain

$$
\frac{d^{2}}{d t^{2}}\left({ }_{c}^{l} R \mathbf{p}_{C}\right)=-\frac{d^{2}}{d t^{2}} \mathbf{p}_{B} \text {. }
$$

Equation (29) shows that aside from a change in sign, the relative acceleration of the ship with respect to the aircraft resolved in $\{I\}$ is equal to the aircraft's inertial acceleration resolved in $\{I\}$. However, in the case of strapdown inertial navigation systems widely in use today [13] the aircraft's inertial acceleration is given in $\{B\}$. Therefore, since

it follows that

$$
\frac{d^{2}}{d t^{2}} \mathbf{p}_{B}={ }_{B}^{I} R^{B} \mathbf{a}
$$

$$
\frac{d^{2}}{d t^{2}}\left({ }_{C}^{1} R \mathbf{p}_{C}\right)=-{ }_{B}^{l} R^{B} \mathbf{a} \text {. }
$$

The nonlinear filters developed in this paper provide estimates of the relative position and velocity of an aircraft with respect to a point on the ship. This information, together with the aircraft's inertial velocity, is sufficient to estimate the ship's inertial velocity and, therefore, its heading. As argued in [14], in the unstructured environment of sea operations the best way to find a ship is by using an IR (infrared) camera. As shown in Figure 2, simple thresholding of an IR image will easily provide information on the coordinates of the centroid of the ship's hottest region (usually its smokestack or boiler room). Therefore, it is only natural that the origin of the ship's coordinate system $\{S\}$ be attached to that point. It is with respect to this same point that the proposed nonlinear filters obtain relative position and velocity. In the immediate vicinity of the ship, where the relative orientation becomes critical, standard structure from motion solutions can be used [15].

We assume that the image of the origin of $\{S\}$ acquired by a camera installed on-board the aircraft is obtained using a simple pinhole camera model of the form [16] (see Figure 3)

$$
\left[\begin{array}{l}
u \\
v
\end{array}\right]=\pi_{f}\left(x_{C}, y_{C}, z_{C}\right)=\frac{f}{x_{C}}\left[\begin{array}{l}
y_{C} \\
z_{C}
\end{array}\right] .
$$

where $f$ is the focal length of the camera and $[u v]^{T}$ are the image 
coordinates of $\mathbf{p}_{C}=\left[\begin{array}{lll}x_{C} & y_{C} & z_{C}\end{array}\right]^{T}$ in the camera's image plane. The natural choice for $[u v]^{r}$ is the centroid of the image points associated with the ship's smokestack. These points can be easily obtained by thresholding from the IR image of a ship, as can be seen in Figure 2.

We also make the following assumptions:

A2 - $x_{C}>0$, that is, the ship is always located in front of the camera's image plane;

$\mathbf{A} 3$ - the rotation matrices and ${ }_{C}^{I} R$ are available from the onboard attitude measurement system.

This assumption is quite reasonable, considering the sophistication achieved by such systems today.

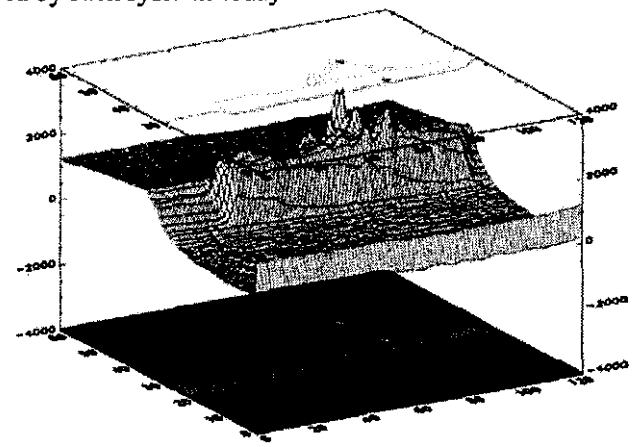

Figure 2: IR image of a ship

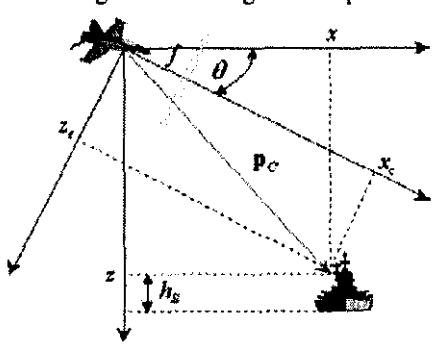

Figure 3: Geometry of the vision/altimeter process model for $\phi=0$

Suppose the aircraft is equipped with a barometric-based sensor that provides a measurement of the altitude of the aircraft with respect to the mean sea level. Assuming the aircraft is sufficiently away from the ship (so as to neglect the height $h_{S}$ of the ship's deck above the mean sea surface), we may assume that

$\mathbf{A 4}-h_{s}=$

Then, using the relation $\quad R \mathbf{p}_{C}$ the altitude measurement

$$
z=g_{\phi, \theta}\left(\mathbf{p}_{C}\right)=-\sin \theta x_{C}-\cos \theta \sin \phi y_{C}+\cos \theta \cos \phi z_{C} .
$$

where $\phi$ and are the roll and pitch angles in the rotation matrix

\section{${ }_{C}^{l} R$ (see Figure 3)}

We now introduce the underlying design model that plays a fundamental role in this paper. Let $\mathbf{y}=[u v z]^{T}$. Then, the model that we consider can be written as

$$
G=\left\{\begin{array}{l}
\dot{\mathbf{p}}=\mathbf{v}, \\
\dot{\mathbf{v}}=-{ }_{B}^{I} R\left({ }^{B} \mathbf{a}_{m}+\mathbf{w}_{a}\right), \\
\mathbf{y}_{m}=g\left(\mathbf{p}_{C}\right)+\mathbf{w}_{y},
\end{array}\right.
$$

where $g\left(\mathbf{p}_{C}\right): \mathfrak{R}^{3} \rightarrow \mathfrak{R}^{3}$ is defined by

$$
\left[\begin{array}{l}
u \\
v \\
z
\end{array}\right]=g\left(\mathbf{p}_{C}\right)=\left[\begin{array}{c}
\pi_{f}\left(\mathbf{p}_{C}\right) \\
g_{\phi_{\theta} \theta}\left(\mathbf{p}_{C}\right)
\end{array}\right] .
$$

and $\mathbf{a}_{m}$ and $\mathbf{y}_{m}$ denote the measured values of $\mathbf{a}$ and $\mathbf{y}$, respectively, the measurements being corrupted by the process noises $\mathbf{w}_{a}$ and $\mathbf{w}_{y}$. In what follows, the deterministic set-up of $H_{\infty}$ filtering [17] will be adopted.

\subsection{Problem definition}

The problem that we consider in this paper consists of determining the relative position and relative velocity of an aircraft with respect to a landing site using vision and other on-board passive sensors. For the sake of clarity, we first tackle the simplified problem of designing a filter with no measurement noise in the model. This exercise is simple, yet it captures some of the key ideas used in the development that follows.

The additional notation that is required is introduced next. We let $\hat{\mathbf{p}}$ and $\hat{\mathbf{v}}$ denote estimates of $\mathbf{p}$ and $\mathbf{v}$, respectively. In the camera frame, they are denoted by $\hat{\mathbf{p}}_{C}, \hat{\mathbf{v}}_{C}$. We assume that the orientation of the camera frame $\{C\}$ with respect to $\{I\}$ is restricted through the set

A5 - $\Lambda_{C}=\left\{\Lambda:|\phi| \leq \phi_{\max },|\theta| \leq \theta_{\max },|\psi| \leq \psi_{\max }\right\}$.

Notice, for example, that $\psi_{\max }$ should be set to $\pi$. We further assume that the vectors $\hat{\mathbf{p}}_{C}$ lie in the compact set

$$
\text { A6- } \begin{aligned}
P_{C}=\left\{\mathbf{p}_{C}: x_{\min } \leq x_{C} \leq x_{\max }, y_{\min }\right. & \leq y_{C} \leq y_{\max }, \\
z_{\min } & \left.\leq z_{C} \leq z_{\max }\right\}
\end{aligned} \text {. }
$$

where $x_{\min }, \ldots, z_{\max }$ are determined from the geometry of the problem at hand. The set $\boldsymbol{P}_{C}$ can be determined as follows. First, compute $P_{C}$ for a nominal orientation of the camera (usually inertial orientation). Determine the maximum range of camera orientation angles with respect to the nominal orientation. Then compute $P_{C}$ by allowing the angles to vary within these predetermined bounds.

In a realistic scenario the image of the ship smokestack will be lost by the onboard camera due, for example, to aircraft rotational motions. This phenomenon is known as an out-of-frame event. Formally, we define a binary signal $s:[0, \infty) \rightarrow\{0,1\}$ :

$$
s(t):= \begin{cases}0 & \text { - out }- \text { of }- \text { frame event at time } t, \\ 1 & \text { - camera tracks the smokestack at time } t .\end{cases}
$$

Furthermore, for a given binary signal $s$ and $t>\tau>0$, let us denote by $T_{s}(\tau, t)$ the amount of time in the interval $(\tau, t)$ that $s=0$. Formally, $T_{s}(\tau, t):=\int(1-s(l)) d l$.

The following assumption plays a crucial role in the development that follows.

A7 - $s$ has brief out-of-frame event, i.e., $T_{s}(, t) \leq T_{0}+\alpha(t-), \quad \forall t \geq \geq 0$, for some $T_{0} \geq 0$, $\alpha \in[0,1]$.

Navigation filter design will aim at ensuring that the estimates $\hat{p}_{C}$ of $\mathbf{p}_{C}$ lie in a compact set 
$\hat{P}_{C}=\left\{\hat{\mathbf{p}}_{C}=\left[\begin{array}{lll}\hat{x}_{C} & \hat{y}_{C} \hat{z}_{C}\end{array}\right]^{T}:\left|\hat{x}_{C}-x_{C}\right| \leq x_{\max }-x_{\min }+d x\right.$,

$\left.\left|\hat{y}_{C}-y_{C}\right| \leq y_{\max }-y_{\min }+d y,\left|\hat{z}_{C}-z_{C}\right| \leq z_{\max }-z_{\min }+d z\right\}$.

where $d x, d y$ and $d z$ are positive numbers, and $d x<x_{\min }$.

F1: Regional Stability. Consider the process model (31) and assume that $\mathbf{w}_{a}=\mathbf{w}_{y}=0$. For a given $\hat{P}_{C}$, find a number $\alpha_{0}>0$, and a dynamical system (filter) $F$ that operates on $\mathbf{y}_{m}$ and $\mathbf{a}_{m}$ to produce estimates $\hat{\mathbf{p}}$ of $\mathbf{p}$, and $\hat{\mathbf{v}}$ of $\mathbf{v}$ in the presence of out-of-frame events, such that:

- $\hat{\mathbf{p}}_{C}(t) \in \hat{P}_{C}$ for any $t>0$,

- $\|\hat{\mathbf{p}}-\mathbf{p}\|+\|\hat{\mathbf{v}}-\mathbf{v}\| \rightarrow 0$ as $t \rightarrow \infty$,

provided that

$$
\left\|\left(\hat{\mathbf{p}}_{C}(0)-\mathbf{p}_{C}(0), \hat{\mathbf{v}}(0)-\mathbf{v}(0)\right)^{T}\right\|<\alpha_{0} .
$$

Notice that the problem described aims at finding a filter that complements the information available from the vision system / barometric pressure sensor with that available from the inertial sensors.

The problem F1 focuses on the stability of the filter. The second filtering problem addresses the scenario where the performance of the filter in the presence of disturbances is considered. F2: Regional Stability and Performance. Consider the process model (31) where ${ }^{1} \mathbf{w}=\left[\mathbf{w}_{a} \mathbf{w}_{y}\right]^{T} \in L_{2}, \| \mathbf{w}_{2} \leq \bar{\omega}$ and let the sets $P_{C}$ and $\hat{P}_{C}$ of allowable position vectors and allowable estimation vectors be defined as above. For given numbers $\gamma>0$ and $\alpha_{0}>0$, find a stable filter $F$ that operates on $\mathbf{y}_{m}$ and $\mathbf{a}_{m}$ to obtain estimates $\hat{\mathbf{p}}$ of $\mathbf{p}, \hat{\mathbf{v}}$ of $\mathbf{v}$ in the presence of out-of-frame events, such that if (33) holds, the filter satisfies the following conditions for all $\mathbf{w} \in L_{2}$ that $\|\mathbf{w}\|_{2} \leq \bar{\omega}$ :

- $\hat{\mathbf{p}}_{C}(t) \in \hat{P}_{C}$ for all $t \geq 0$,

- $\|\hat{\mathbf{p}}-\mathbf{p}\|+\|\hat{\mathbf{v}}-\mathbf{v}\| \rightarrow 0$ as $t \rightarrow \infty^{2}$,

- $\left\|T_{e w}\right\|_{2, i}<\gamma$, where $\mathbf{e}:=\hat{\mathbf{p}}-\mathbf{p}$ is the estimation error and $T_{e w}: \mathbf{w} \rightarrow \mathbf{e}$.

Notice the technical requirement that an allowable set of position estimates $\hat{P}_{C}$ be specified. As is shown later, this requirement is essential to establishing the boundedness of a certain operator for all possible values of the estimates $\hat{\mathbf{p}}$. In practice, the "size" of the allowable region $\hat{P}$ plays the role of a design parameter.

\subsection{Proposed solution}

This section describes the solutions to problems F1 and F2. First, however, we need the following basic results. Let $H$ denote the Jacobian of $g\left(\mathbf{p}_{C}\right)$ with respect to $\mathbf{p}_{C}$. From the definition of $g\left(\mathbf{p}_{C}\right)$, it follows that

${ }^{1}$ Given a signal $z$ we denote by $\|z\|_{2}$ the $L_{2}$-norm of $z$, i.e., $\|z\|_{2}=\sqrt{\int_{0}^{\infty}\|z(t)\|^{2} d t}$.

${ }^{2}$ As long as $\mathbf{w} \in L_{2}$ we always get convergence to zero.

$$
H\left(\mathbf{p}_{C}\right)=\left[\begin{array}{ccc}
-f y_{C} x_{C}^{-2} & f x_{C}^{-1} & 0 \\
-f z_{C} x_{C}^{-2} & 0 & f x_{C}^{-1} \\
-\sin \theta & -\cos \theta \sin \phi & \cos \theta \cos \phi
\end{array}\right]
$$

It is easy to check that $\operatorname{det}(H)=f^{2} x_{C}^{-3} z_{C}$. Therefore, $H$ is not invertible if and only if $z=0$. This implies that $H\left(\mathbf{p}_{C}\right)$ is invertible for all admissible values of $\mathbf{p}_{C}, \phi$, and $\theta$.

The next result is adopted from [5] and plays a key role in the development that follows. In particular, identity (35) makes it possible to show that the proposed nonlinear filter error dynamics represent an LPV system. This leads to the utilization of the LPV framework to reduce the estimation problem to that of determining the feasibility of a set of LMI's (see proofs of Theorems 3.3 and 3.4).

Lemma 3.1 Let $g\left(\mathbf{p}_{C}\right)$ be given by equation (32). Then

$$
g\left(\hat{\mathbf{p}}_{C}\right)-g\left(\mathbf{p}_{C}\right)=L\left(\hat{\mathbf{p}}_{C}, \mathbf{p}_{C}\right) H\left(\hat{\mathbf{p}}_{C}\right)\left(\hat{\mathbf{p}}_{C}-\mathbf{p}_{C}\right),
$$

where His given in equation (34), $\hat{\mathbf{p}}_{C}=\left[\hat{x}_{C} \hat{y}_{C} \hat{z}_{C}\right]^{T}$ and

$$
L\left(\hat{\mathbf{p}}_{C}, \mathbf{p}_{C}\right)=\left[\begin{array}{ccc}
\hat{x}_{C} x_{C}^{-1} & 0 & 0 \\
0 & \hat{x}_{C} x_{C}^{-1} & 0 \\
0 & 0 & 1
\end{array}\right]
$$

Lemma 3.2 Let $\varphi: \mathfrak{R}^{6} \rightarrow \mathfrak{R}^{3 \times 3}$, and $\varphi_{1}: \mathfrak{R}^{3} \rightarrow \mathfrak{R}^{3 \times 3}$ be the operators defined by $\varphi\left(\hat{\mathbf{p}}_{C}, \mathbf{p}_{C}\right)=H^{T}\left(\hat{\mathbf{p}}_{C}\right) L\left(\hat{\mathbf{p}}_{C}, \mathbf{p}_{C}\right) H\left(\hat{\mathbf{p}}_{C}\right)$ and $\varphi_{1}\left(\hat{\mathbf{p}}_{C}\right)=H^{T}\left(\hat{\mathbf{p}}_{C}\right) H\left(\hat{\mathbf{p}}_{C}\right)$. Then $\varphi\left(\hat{\mathbf{p}}_{C}, \mathbf{p}_{C}\right)>0$, $\varphi_{1}\left(\hat{\mathbf{p}}_{C}\right)>0, \forall \hat{\mathbf{p}}_{C} \in P_{C}$ and $\hat{\mathbf{p}}_{C} \in \hat{P}_{C}$.

Proof: The proof follows directly from assumptions $\mathbf{A 1 - A 3}$ and the definitions of $H$ and $L$.

The following result provides a solution to problem $\mathbf{F 1}$.

Theorem 3.3 Let $\hat{P}_{C}$ be given and assume that AI-A7 hold and $r_{x}=\frac{x_{\max }-x_{\min }+d x}{x_{\min }}<1$. Suppose there exists a matrix $X=X^{T} \in \mathfrak{R}^{6 \times 6}$ and positive constants $\alpha, \alpha_{0}, \lambda_{0,} \mu, T_{0}$, such that $\alpha<\alpha^{*}$ and

$$
\begin{array}{r}
X>0, \\
F^{T} X+X F-2\left(1-r_{x}\right)^{2} \varepsilon C^{T} C \leq-\lambda_{0} X, \\
F^{T} X+X F \leq \mu X, \\
X-\delta^{-2} C^{T} C \geq 0, \\
\alpha_{0}^{-2} e^{-\left(\lambda_{0}+\mu\right) T_{0}} I-X \geq 0,
\end{array}
$$

where $F:=\left[\begin{array}{ll}0 & I \\ 0 & 0\end{array}\right], C:=\left[\begin{array}{ll}I & 0\end{array}\right]$, and

$$
\delta:=\min \left\{x_{\max }-x_{\min }+d x, y_{\max }-y_{\min }+d y, z_{\max }-z_{\min }+d z\right\} \text {, }
$$$$
\varepsilon: \min _{\hat{\mathbf{p}}_{C} \hat{P}_{C}} \min \left(H^{T}\left(\hat{\mathbf{p}}_{C}\right) H\left(\hat{\mathbf{p}}_{C}\right)\right) .
$$

Define a filter (see Figure 4)

$$
\begin{aligned}
& \left\{\dot{\hat{\mathbf{p}}}=\hat{\mathbf{v}}+s K_{1}{ }_{C}^{l} R H^{T}\left(\hat{\mathbf{p}}_{C}\right)\left(g\left(\hat{\mathbf{p}}_{C}\right)-\mathbf{y}_{m}\right),\right. \\
F_{1}:= & \left\{\dot{\hat{\mathbf{v}}}=-{ }_{B}^{I} R^{B} \mathbf{a}_{m}+s K_{2}{ }_{C}^{l} R H^{T}\left(\hat{\mathbf{p}}_{C}\right)\left(g\left(\hat{\mathbf{p}}_{C}\right)-\mathbf{y}_{m}\right),\right. \\
& {\left[\hat{\mathbf{p}}_{C}={ }_{I}^{C} R \hat{\mathbf{p}},\right.}
\end{aligned}
$$

where 
$s(t):= \begin{cases}0 & - \text { out }- \text { of }- \text { frame event at time } t, \\ 1 & \text { - camera tracks the smokestack at time } t,\end{cases}$

and

$$
\left[\begin{array}{l}
K_{1} \\
K_{2}
\end{array}\right]=-X^{-1}\left(1-r_{x}\right) C^{r}
$$

Then the filter $F_{1}$ solves the filtering problem $F$ 1, provided that (33) holds.

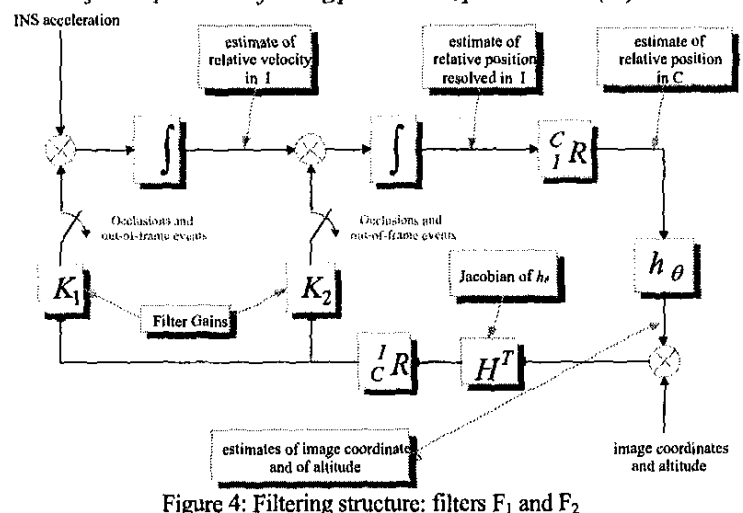

Proof: Define the error state $e_{1}:=\hat{\mathbf{p}}-\mathbf{p}$ and $\mathbf{e}_{2}:=\hat{\mathbf{v}}-\mathbf{v}$. Let $\mathbf{e}=\left[\begin{array}{ll}\mathbf{e}_{1}^{T} & \mathbf{e}_{2}^{T}\end{array}\right]^{T}$. Then, using Lemma 3.1 and simple algebra it can be shown that the error dynamics can be written as

$$
\frac{d}{d t}\left[\begin{array}{l}
\mathbf{e}_{1} \\
\mathbf{e}_{2}
\end{array}\right]=\left(F+s K_{C}^{I} R \phi\left(\hat{\mathbf{p}}_{C}, \mathbf{p}_{C}\right){ }_{C}^{J} R^{T} C\right)\left[\begin{array}{l}
\mathbf{e}_{1} \\
\mathbf{e}_{2}
\end{array}\right],
$$

where $K:=\left[K_{1}^{T} K_{1}^{T}\right]^{T}$. Notice that the error dynamics given by (44) represent an LPV system that depends on $\left(\mathbf{p}_{c}, \mathbf{e}_{1}, s\right)$. Now, to show that $\hat{\mathbf{p}}_{c} \in \hat{P}_{C}$, it is sufficient to show that $\left\|\mathbf{e}_{1}\right\| \leq \delta$, or equivalently that $\left[\mathbf{e}_{1}^{T} \mathbf{e}_{2}^{T}\right]^{T}$ remains in $\Omega:=\{\mathbf{e}\|C \mathrm{e}\| \leq \delta\}$. From Corollary 2.2 and $\mathbf{A 7}$, we conclude that this is true provided that there exists a matrix $X>0$ and constants $\lambda_{0}, \mu$, such that (38) and (39) hold,

$$
\begin{array}{r}
\left(F+K_{C}^{l} R \varphi\left(\hat{\mathbf{p}}_{C}, \mathbf{p}_{C}\right)_{C}^{l} R^{T} C\right)^{T} X+ \\
X\left(F+K_{C}^{I} R \varphi\left(\hat{\mathbf{p}}_{C}, \mathbf{p}_{C}\right)_{C}^{I} R^{T} C\right) \leq-\lambda_{0} X
\end{array}
$$

for all times for which $\left[\mathbf{e}_{1}^{T} \mathbf{e}_{2}^{T}\right]^{r} \in \Omega$, and $s=1$, and

$$
\left[\mathbf{e}(0)_{1}^{T} \mathbf{e}(0)_{2}^{T}\right] X\left[\mathbf{e}(0)_{1}^{T} \mathbf{e}(0)_{2}^{T}\right]^{T} \leq e^{-\left(\lambda_{0}+\mu\right) T_{0}} .
$$

Inequality (46) follows from $\left\|\left[\mathbf{e}(0)_{1}^{T} \mathbf{e}(0)_{2}^{T}\right]\right\| \leq \alpha_{0}$ and (40). In the following, we focus on the solvability of (45). Prom (43), we conclude that (45) is equivalent to

$$
F^{T} X+X F+\left[\begin{array}{ccc}
-2\left(1-r_{x}\right)_{C}^{I} R H^{T}\left(\hat{\mathbf{p}}_{C}\right) L H\left(\hat{\mathbf{p}}_{C}\right)_{C}^{I} R^{T} & 0 \\
) & 0 & 0
\end{array}\right](47)
$$

In the set $\Omega,\left\|\mathrm{e}_{1}\right\| \leq \delta$, so

$$
L:=I+\left[\begin{array}{ccc}
\hat{x}_{C}-x_{C} x_{C}^{-1} & 0 & 0 \\
0 & \left.\hat{x}_{C}-x_{C}\right) x_{C}^{-1} & 0 \\
0 & 0 & 1
\end{array}\right]>1-r_{x}
$$

Therefore,

$$
\begin{array}{r}
-2\left(1-r_{x}\right)_{C}^{l} R H^{T}\left(\hat{\mathbf{p}}_{C}\right) L H\left(\hat{\mathbf{p}}_{C}\right)_{C}^{l} R^{T} \\
<-2\left(1-r_{x}\right)_{C}^{2} R H^{T}\left(\hat{\mathbf{p}}_{C}\right) H\left(\hat{\mathbf{p}}_{C}\right)_{C}^{I} R^{T}<-2\left(1-r_{x}\right)^{2} \varepsilon I
\end{array}
$$

Because of this and the fact that $C^{T} C=\left[\begin{array}{ll}I & 0 \\ 0 & 0\end{array}\right]$, we conclude that (37) implies (47) in the set . Now, from Corollary 2.2 it follows that $\left\|\mathrm{e}(t)_{1}\right\| \leq \delta, \quad \forall t \geq 0$ and $\mathbf{e}_{1}(t), \mathbf{e}_{2}(t) \rightarrow 0$ as $t \rightarrow \infty$.

The solvability of the inequality (37) is addressed in [10]. There, it is shown that the inequality has a solution if and only if $r_{x}<1$. The next theorem provides a solution to the filtering problem $\mathbf{F 2}$

Theorem 3.4 Let $\hat{P}_{\mathrm{C}}$ be given and assume that A1-A 7 hold and $r_{x}=\frac{x_{\max }-x_{\min }+d x}{x_{\min }}<1$. For a given gain $\gamma>0$, suppose there exists a matrix $X=X^{T} \in \mathfrak{R}^{6 \times 6}$ and positive constants , ${ }_{0}, \lambda_{0,}, \mu$, $T_{0,}$ such that $\alpha<\alpha^{*}$ and

$X>0$,

$$
\begin{array}{r}
F^{T} X+X F+X F^{T} F X+\frac{e^{\left(\hat{\lambda}_{0}+\mu\right) T_{0}}}{\lambda \gamma^{2}} C^{T} C \leq \mu X \\
X-\delta^{-2} C^{T} C \geq 0, \\
\left.\left.\alpha_{0}^{-2} e^{-\left(\lambda_{0}\right.} \quad\right) \pi_{0}-\bar{\omega}^{2}\right) I-X \geq 0,
\end{array}
$$

where $\left.F:=\left[\begin{array}{ll}0 & I \\ 0 & 0\end{array}\right], C:=I \quad 0\right], \varepsilon:=\min _{\hat{\mathbf{p}}_{C} \in \hat{P}_{C}} \lambda_{\min }\left(\varphi_{1}\left(\hat{\mathbf{p}}_{C}\right)\right)$, and $\delta:=\min \left\{x_{\max }-x_{\min }+d x, y_{\max }-y_{\text {min }}+d y, z_{\text {max }}-z_{\text {min }}+d z\right\}$. Define a filter (see Figure 4)

$$
F_{2}:=\left\{\begin{array}{l}
\not{\mathbf{p}}=\hat{\mathbf{v}}+s K_{1}{ }_{C}^{I} R H^{T}\left(\hat{\mathbf{p}}_{C}\right)\left(g\left(\hat{\mathbf{p}}_{C}\right)-\mathbf{y}_{m}\right), \\
\forall=-{ }_{B}^{l} R^{B} \mathbf{a}_{m}+s K_{2}{ }_{C}^{I} R H^{T}\left(\hat{\mathbf{p}}_{C}\right)\left(g\left(\hat{\mathbf{p}}_{C}\right)-\mathbf{y}_{m}\right), \\
\hat{\mathbf{p}}_{C}={ }_{I}^{C} R \hat{\mathbf{p}},
\end{array}\right.
$$

where

$$
\left[\begin{array}{l}
K_{1} \\
K_{2}
\end{array}\right]:=-X^{-1}\left(1-r_{x}\right) C^{T}
$$

Then the filter $F_{2}$ solves the filtering problem $F 2$ if $(33)$ holds.

Proof: Define the error state $\mathbf{e}_{1}:=\hat{\mathbf{p}}-\mathbf{p}$ and $\mathbf{e}_{2}:=\hat{\mathbf{v}}-\mathbf{v}$.

Then, the error dynamics admit the state-space realization

$$
\begin{aligned}
& \frac{d}{d t}\left[\begin{array}{l}
\left.\mathbf{e}_{1}\right] \\
\mathbf{e}_{2}
\end{array}\right]=\left(F+s K_{C}^{I} R \quad\left(\hat{\mathbf{p}}_{C}, \mathbf{p}_{C}\right)_{C}^{I} R^{T} C\right)\left[\begin{array}{l}
\mathbf{e}_{1} \\
\mathbf{e}_{2}
\end{array}\right] \\
& +\left[\left[\begin{array}{c}
0 \\
\left.{ }_{B}{ }_{B} R\right]
\end{array}\right]-s K_{C}^{I} R H^{T}\left(\hat{\mathbf{p}}_{\mathrm{C}}\right){ }_{\rfloor}^{\top} \mathbf{w},\right.
\end{aligned}
$$

where $\left.\mathbf{w}: \quad \mathbf{w}_{a}^{T} \mathbf{w}_{y}^{T}\right]^{T}$ and $K:\left[K_{1}^{T} K_{1}^{T}\right]^{T}$. We now show that if the inequalities (50)-(54) are satisfied, then $\hat{\mathbf{p}}_{C} \in \hat{P}_{C}$ for all $\mathbf{w} \in L_{2},\|\mathbf{w}\|_{2} \leq \overline{\boldsymbol{o}}$, when (33) holds. 
To prove that $\hat{\mathbf{p}}_{C} \in \hat{P}_{C}$, it is sufficient to show that $\left\|\mathbf{e}_{1}\right\| \leq \delta$, or equivalently that $\left[\mathbf{e}_{1}^{T} \mathbf{e}_{2}^{T}\right]^{T}$ remains in $\Omega:=\{\mathbf{e}\|C \mathrm{e}\| \leq \delta\}$. From Corollary 2.4 (with $\rho:=\gamma \sqrt{\frac{\lambda}{e^{\left(\lambda_{0}+\mu\right) T_{0}} \lambda_{0}}}$ ) and A7, we conclude that this is true provided that there exists a matrix $X>0$ and constants $\lambda_{f,}, \mu$ such that (52) and (53) hold,

$$
\begin{aligned}
& \left(F+K_{C}^{I} R \varphi\left(\hat{\mathbf{p}}_{C}, \mathbf{p}_{C}\right)_{C}^{I} R^{T} C\right)^{T} X+ \\
& \quad X\left(F+K_{C}^{I} R \varphi\left(\hat{\mathbf{p}}_{C}, \mathbf{p}_{C}\right)_{C}^{I} R^{T} C\right) \leq-\lambda_{0} X
\end{aligned}
$$

for all times for which $\left[\mathrm{e}_{1}^{T} \mathbf{e}_{2}^{T}\right]^{T} \in \Omega$, and $s=1$, and

$$
\left[\mathbf{e}(0)_{1}^{T} \mathbf{e}(0)_{2}^{T}\right] X\left[\mathbf{e}(0)_{1}^{T} \mathbf{e}(0)_{2}^{T}\right]^{T}+\int_{b}^{\infty}\|\mathbf{w}(s)\|^{2} d s \leq e^{-\left(\lambda_{0}+\mu\right) T_{0}} .
$$

In (52) and to use (58) we used the fact that

$$
\left[\begin{array}{ll}
0 & 0 \\
{ }_{B}^{I} R & 0
\end{array}\right]\left[\begin{array}{cc}
0 & { }_{B}^{I} R \\
0 & 0
\end{array}\right]=\left[\begin{array}{cc}
0 & 0 \\
0 & I
\end{array}\right]=F^{T} F \text {. }
$$

Inequality (59) follows from $\|\left[\mathrm{e}(0)_{1}^{T} \mathrm{e}(0)_{2}^{T}\left\|\leq \alpha_{0},\right\| \mathrm{w} \|_{2} \leq \bar{\omega}\right.$, and (54). In the following, we focus on the solvability of (58). Because

$$
+\left[\begin{array}{rr}
\frac{e^{\left(\lambda_{0}+\mu\right) T_{0}} \lambda_{0}}{\lambda \gamma^{2}} I+\left(1-r_{x}\right)^{2}{ }_{C}^{I} R \varphi_{1}{ }_{C}^{I} R^{T}-2\left(1-r_{x}\right)_{C}^{I} R \varphi_{C}^{I} R^{T} & 0 \\
0 & 0
\end{array}\right] .
$$

Because of the first inequality in (49), this inequality holds

$$
+\left[\begin{array}{rr}
\frac{e^{\left(\lambda_{0} \mu\right) T_{0}} \lambda_{0}}{\lambda \gamma^{2}} I-\left(1-r_{x}\right)^{2}{ }_{C}^{I} R \varphi_{1 C}{ }^{I} R^{T} & 0 \\
0 & 0
\end{array}\right] \leq-\lambda_{0} X
$$

and then, using the second inequality in (49), we further conclude

Here we used the fact that $C^{T} C=\left[\begin{array}{ll}I & 0 \\ 0 & 0\end{array}\right]$. From Schur complements [7] and the definition (41), (60) holds because of (51). The theorem then follows from Corollary 2.4 .

The next theorem derives necessary and sufficient conditions under which $(51)$ is satisfied.

Theorem 3.5 Let $F$, and $\varepsilon$ and be defined in Theorem 3.4. Then $\exists X=X^{T}>0$ such that

$$
\begin{aligned}
& {\left[F^{T} X+X F+\lambda_{0} X+\left(\frac{\left.e^{\left(\lambda_{0}\right.}\right)^{T} \lambda_{0}}{\lambda \gamma^{2}} I-\left(1-r_{x}\right)^{2}\right) C^{T} C \quad X F^{T}\right] 0} \\
& \Leftrightarrow \frac{e^{\left(\lambda_{0}\right) T_{0}} \lambda_{0}}{\lambda \gamma^{2}}-\left(1-r_{x}\right)^{2} \leq 0 .
\end{aligned}
$$

Proof: Follows by rescaling in the proof of Theorem 4.5 in [10].

Remark 3.1 Theorem 3.5 shows that the LMI (51) is feasible if and only if

$$
\left.\frac{e^{\left(\lambda_{0}\right) T_{0}} \lambda_{0}}{\lambda \gamma^{2}} I-\left(1-r_{x}\right)^{2} \leq 0 \Leftrightarrow \gamma^{2} \geq \frac{e^{\left(\lambda_{0}\right) T_{0}} \lambda_{0}}{\lambda\left(1-r_{x}\right)^{2}} . \quad\right\}
$$

Recall that

Therefore, we obtain

$$
\gamma^{2} \geq \frac{e^{\left(\lambda_{0}\right.}{ }^{T_{0}} \lambda_{0}}{\lambda\left(1-r_{x}\right)^{2}}=\max _{\mathbf{p}_{C} \in P_{C}}\left\|H^{T}\left(\mathbf{p}_{C}\right) H_{T}\left(\mathbf{p}_{C}\right)^{-1}\right\| .
$$

This inequality imposes a lower bound on the achievable values of $\gamma$. Furthermore, since $\lambda:=\lambda_{0}-\alpha\left(\lambda_{0}+\mu\right)$, it follows that

$$
\lim _{T_{0} \rightarrow 0, a \rightarrow 0} \frac{e^{\left(\lambda_{0}\right) T_{0}} \lambda_{0}}{\lambda\left(1-r_{x}\right)^{2}}=\frac{1}{\left(1-r_{x}\right)^{2}} .
$$

The above expression shows that the lower bound on the achievable $\gamma$ in the absence of out of frame events converges to the lower bound derived in [10].

The bound derived in (61) is similar to the classical Positional Dilution of Precision (PDOP) metric that is commonly used in navigation systems to determine a lower bound on the achievable error covariance as a function of geometry of the underlying navigation problem $[20,21,13]$. Using our notation, the classical PDOP can be written as

$$
P D O P=\sqrt{\operatorname{tr}\left(H^{T}\left(\mathbf{p}_{C}\right) H\left(\mathbf{p}_{C}\right)\right)^{l}} .
$$

We therefore see that the new bound derived in this paper captures a worst case performance scenario and the estimate of $x_{C}$ increases the lower bound on the achievable $\gamma$, since $1>\left(1-r_{x}\right)^{2}>0$.

Remark 3.2 The filters used in this paper borrowed from the structure of the nonlinear observer proposed in [5]. Both filters are designed for a process model that exhibits linear dynamics and nonlinear measurement equations. In view of this fact, one is naturally driven to ask the following question: why not simply solve the measurement equation to obtain estimate of $\mathbf{p}_{C}$ that can in turn drive a linear filter with a much simpler structure? This technique was, in fact, applied in an earlier version of the work reported in [5]. However, as pointed out by the authors the latency inherent to this approach led to unacceptable results. This stemmed from the fact that the estimate of $\mathbf{p}_{C}$ obtained by the nonlinear solver from the measurement equation and used by the linear filter represented a "delayed version" of the true position.

Furthermore, the algorithm used by the nonlinear solver requires inverting the Jacobian. In a noisy environment this may lead to excessive noise amplification. This problem is entirely avoided by the filters proposed in this paper as well as by the nonlinear observer in [5]. Finally, the gains used by every filter in this paper are of the form similar to the gains of optimal filters obtained for the linear time invariant (LTI) case. This is important, since in the LTI case even if the output matrix is invertible the optimal gain does not require inversion of this matrix.

3.5 Numerical implementation and performance studies In the absence of out-of-frame events $\left(\alpha=0, T_{0}=0\right)$ the matrix inequalities developed in Theorem 3.4 can be reduced to the following form: 


$$
\left[\begin{array}{rr}
F^{T} X+X F+\left(\frac{1}{\gamma^{2}}-\left(1-r_{x}\right)^{2} \varepsilon\right) C^{T} C & X F^{T} \\
F X & -I
\end{array}\right]^{<0},
$$

where $\varepsilon$ is inversely proportional to the size of $\hat{P}_{C}, \gamma$ determines the filter's performance and $\alpha_{0}$ - the bound on the initial error in position and velocity estimates. Clearly from the design standpoint, one would like to minimize $\varepsilon, \gamma$ and maximize $\alpha_{0}$.

Let $w_{1}=\gamma^{2}, w_{2}=\alpha_{0}^{-2}, w_{3}=\varepsilon$. Define the cost functional $J=c_{1} w_{1}+c_{2} w_{2}+c_{3} w_{3}$, where $c_{l}, c_{2}, c_{3}$ are positive weights to be selected by the designer. Now the design problem discussed above can be reduced to the following convex optimization problem:

find $\min J$ subject to

$$
\begin{aligned}
& {\left[\begin{array}{ccc}
F^{r} X+X F-\left(1-r_{x}\right)^{2} w_{3} C^{T} C & C^{T} & X F^{T} \\
C & -w_{1} & 0 \\
X F & 0 & -I
\end{array}\right]<0,} \\
& w_{2}\left(1-\bar{\omega}^{2}\right)-X>0 \text {, } \\
& X-\delta^{-2} C^{T} C>0 .
\end{aligned}
$$

This optimization problem was solved numerically using MATLAB's LMI toolbox [9]. The resulting values of $X, \alpha_{0}$, and $\gamma$ where then used to study the impact of the out-of-frame events on the filter performance. For example, in the absence of out-of-frame events the value of the performance bound $\gamma$ achieved by the filter was 35 . However, in the presence of out-of-frame events, as discussed above, the value of $\gamma$ increases as a function of $T_{0}$ as illustrated in Figure 5. (Recall that we assumed that on any finite interval $t$ $\tau, t>\tau$ the duration of an out-of-frame event is bounded above by $T_{0}+\alpha(t-\tau), \alpha \in[0,1]$.) Since the numerical values of $\alpha$ obtained were on the order of $10^{-5}-10^{-4}$ their impact on the levels of achievable $\gamma$ was negligible. Furthermore, as the graph in Figure 5 suggests $T_{0}$ exhibits logarithmic dependence on $\gamma$. This implies that for values of $T_{0}>2.5 \mathrm{sec}$, small increases in $T_{0}$ result in large increases in achievable $\gamma$, i.e. the filter performance deteriorates rapidly once $T_{0}$ passes this threshold.

Another interesting trade-off is shown in Figure 6, where for two value pairs of $\left(T_{0}, \gamma\right)=(0.52,55),\left(T_{0}, \gamma\right)=(2.5,250)$ the graphs of $\bar{\omega}$ v.s. $\alpha_{0}$ are plotted. Recall, in this paper $\alpha_{0}$ defines the bound on the norm of the initial estimation error (33), while $\bar{\omega}$ defines the bound on the norm of the sensor noise. Figure 6 shows the trade-off between the size of the initial estimation error tolerated by the filter and the bound on the sensor noise. Clearly, as $T_{0}$ increases the achievable values of $\bar{\omega}$ and $\alpha_{0}$ decrease.

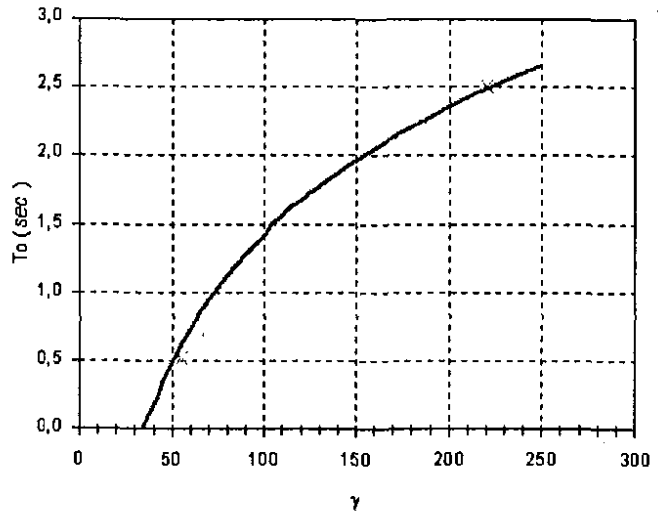

Figure 5: Achievable $\gamma$ versus $T_{0}$

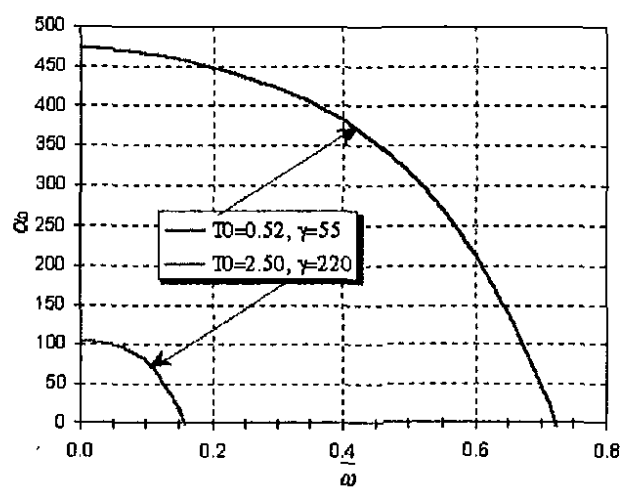

Figure 6: Achievable $\alpha_{0}$ versus $\bar{\omega}$

\subsection{Experimental setup and flight-test results}

This section describes the experimental setup and the flight test experiments that were performed to test the performance of the nonlinear filter obtained in the previous section. The Frog UAV operated by the controls lab at NPS was equipped with an Infrared video camera. The camera included a Boeing U3000A uncooled 8-12 microns (micrometers). The pixel resolution of the camera was $320 \times 240$. The UAV was also equipped with a Trimble AgGPS 132 Differential Global Positioning System (DGPS). An illustration of the flight test setup is provided in Figure 7.

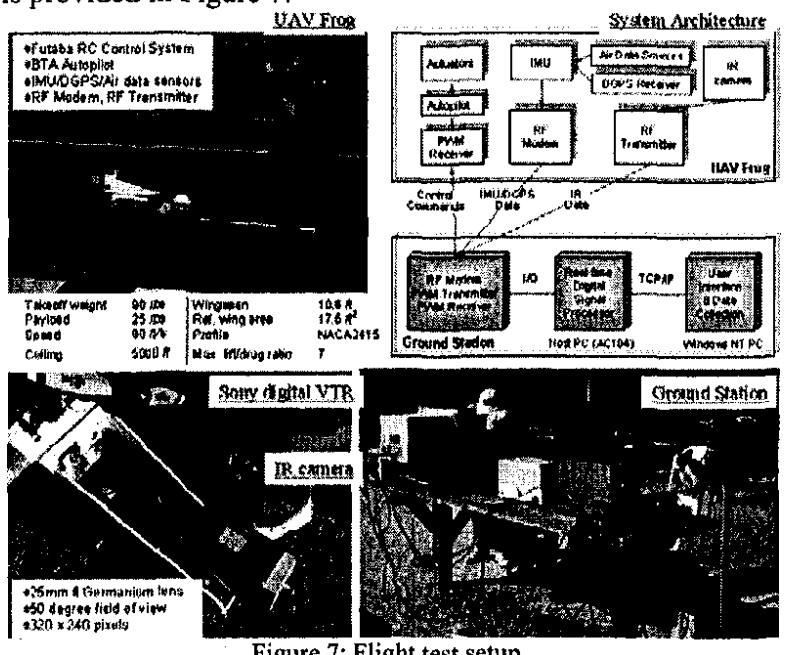

Figure 7: Flight test setup 
Flight tests were conducted at Camp Roberts air field operated by NFS. Charcoal grills were used to model the hot spots on the ship (see Figure 8). Samples of UAV trajectories recorded by onboard DGPS are shown in Figure 9. Similarly, samples of IR images collected by the onboard IR camera are

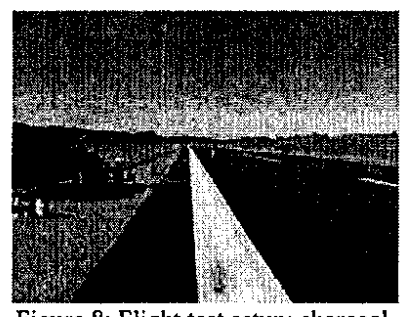

Figure 8: Flight test setup: charcoal grills

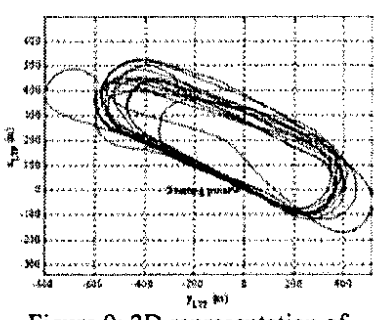

Figure 9: 2D representation of DGPS-recorded trajectories
The image processing problem, i.e. that of finding the hot spots in the image on the runway, turned out to be nontrivial due to the presence of multiple hot spots in the surrounding area. This is In contrast to finding hot spots on a ship, where they are clearly much hotter than the ocean (see Figure 11).
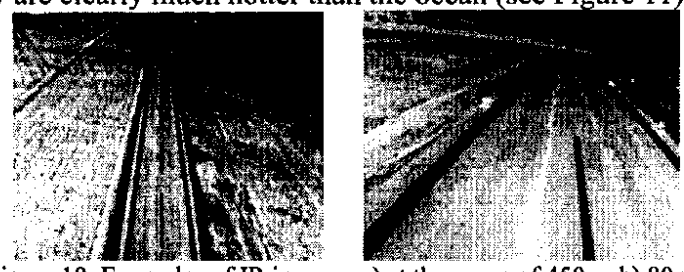

Figure 10: Examples of IR images: a) at the range of $450 \mathrm{~m}$, b) $80 \mathrm{~m}$

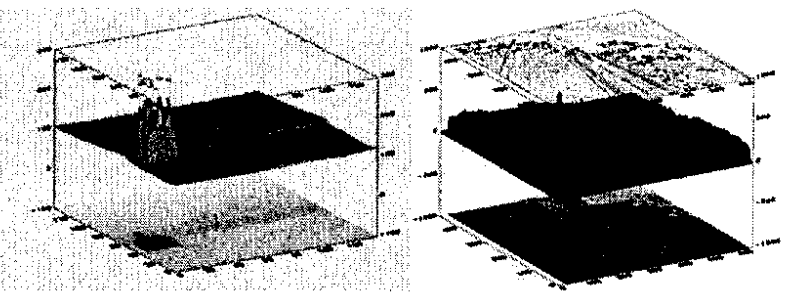

Figure 11: Comparisons of IR images: $a$ ) of a ship and b) of the hot spots

As a result an image-processing algorithm was developed to find and track the hot spots observed by the IR camera onboard the UAV Frog. The algorithm consisted of two steps. The first step included finding the hot spots in the initial image and involved a search over the complete image plane (see Figure 12). Once the hot spots were found in the initial image, they were tracked for the remainder of the approach (see Figure 12). The critical element of this second step was reliance on the inertial data to predict the approximate location of the hot spots in the next image and to recover from the out-of-frame events.

The image plane coordinates and GPS altitude were used by the integrated IR/Inertial filter to compute relative position and velocity with respect to the nearest hot spot. Figure 13 shows the results of applying the integrated IR/Inertial filter to the flight test data. In particular, the upper graph shows the DGPS landing approach trajectory. The bottom left graph shows the estimation errors computed by comparing the DGPS position with the position estimates produced by the filter. Finally, the bottom right graph shows the response of the filter to an out of frame event. Clearly, the filter performed well.

\section{Conclusions}

This paper introduced the concept of LPV systems with brief instabilities and derived new results for stability and performance analysis of such systems, where performance is evaluated in terms of $L_{2}$ induced norms. The main results show that stability and performance can be assessed by examining the feasibility of parameterized sets of LMIs. These results were applied to the design of an integrated vision/inertial navigation filter with guaranteed stability and performance id the presence of out-of-frame events. Numerical trade-off studies were conducted to determine filter's achievable performance versus the duration of the out-of-frame events. Finally, the filter was tested using flight test data collected by a UAV equipped with inertial sensors and IR camera. The results of the test showed the filter to perform well in the presence of out-offrame events.

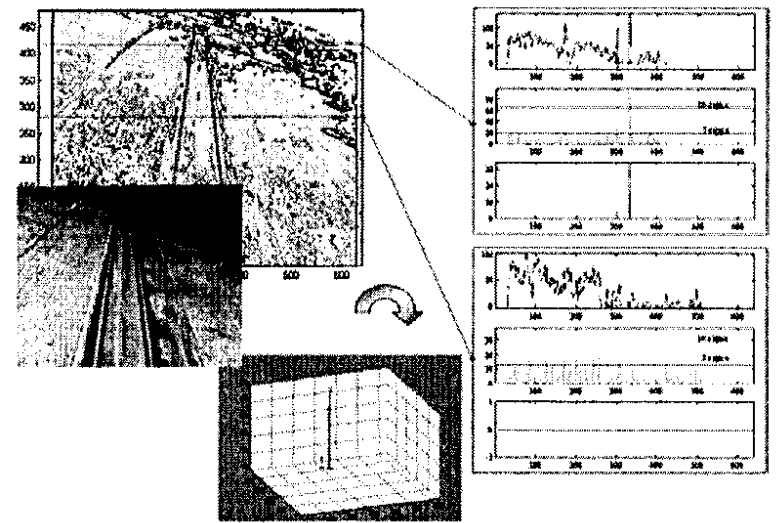

Figure 12: Main ideas of the first step for IR image processing

Figure 13: Filter's performance during final approach

\section{References}

[1] Y. Bresle and S. Merhav, "On-line vehicle motion estimation from visual terrain information. Part II: Ground velocity and position estimation," IEEE Transactions on Aeraspace and Electronic Systems, Vol.22, No.5, September 1986,pp. 588-604.

[2] Y. Bresle and S. Merhav, "Recursive image registration with application to motion estimation," IEEE Transactions on Acoustic, Speech, and Signal Processing, Vol.35, No.1, January 1987, pp. 70-85.

[3] G. Chatterji, P. Menon, and B. Shridar, "GPS/Machine vision navigation system for aircraft," IEEE Transactions on Aerospace and Electronic Systems, Vol.33, No.3, July 1997, pp. 1012-1025.

[4] G. Chatterji, P. Menon, and B. Shridar, "Vision based position and attitude determination for aircraft night landing," AIAA Journal of Guidance, Control and Dynamics, Vol.21, No.1, January-February 1998, pp. 84-92.

[5] A. Rizzi and D. Koditscheck, "An active visual estimator for dexterous manipulation," IEEE Transactions on Robotics and Automation, Vol.12, No.5, October 1996, pp. 697-713.

[6] G. Becker and A. Packard, "Robust performance of linear, parametrically varying systems using parametrically- dependent linear, dynamic feedback," Systems and Control Letters, 1994. 
[7] S. Boyd, L. El Ghaoui, E. Feron and B. Balakrishnan. Linear Matrix Inequalities in Systems and Control Theory. SIAM Studies in Appliod Mathematics, Philadelphia, 1994.

[8] M. Green and D. Limebeer. Linear Robust Control. Prentice-Hall, 1995.

[9] P.Gahinet, A.Nemirovski, AJ. Laub, M. Chilali, LMI Control Toolbox User's Giide. The Math Works Inc., 1997.

[10] I. Kaminer, W. Kang, O. Yakimenko and A. Pascoal, "Design of Integrated Vision/Inertial Navigation Systems using Nonlinear Filtering," IEEE Transactions on Aeraspace and Electronic Systems, Vol..37, No.1, January 2001, pp. 158172.

[11] C. Scherer, "Generalized Mixed $\mathrm{H}_{2} / \mathrm{H}_{\infty}$ Synthesis for Linear Parameter Varying Systems," preprint, 1997

[12] K. Britting. Inertial Navigation System Analysis. Wiley-Interscience, 1971.

[13] G. Siouris. Aerospace Avionics Systems: a Modern Synthesis. Academic Press Inc., 1993.

[14] W. J. Lentz, NPS Internal Comespondence, 1999.

[15] J. Weng, T. S. Huang, and N. Ahuja. Motion and Structure from Image Sequences. Springer-Verlag Series in Information Sciences, 1993.

[16] G. Hager, "A modular System for Robust Positioning using Feedback from Stereo Vision," IEEE Transactions on Robotics and Automation, Vol.13, No.4, August 1997, pp. 582-595.

[17] K. Nagpal and P. Khargonekar, "Filtering and Smoothing in an $\mathbf{H}_{\infty}$ setting," IEEE Transactions on Automatic Control, Vol.36, 1991, pp.152166.
[18] R. Brown, "Integrated Navigation Systems and Kalman Filtering: a Perspective," Joumal of the Institute of Navigation, Winter 1972-73, Vol.19, No.4 pp.355-362.

[19] S. Merhav. Aerospace Sensor Systems and Applications. SpringerVerlag, 1996.

[20] P. Massatt and K. Rundick, "Geometric Formulas for Dilution of Precision Calculations," Joumal of Institute of Navigation, Vol.37, No.4, Winter 199091, pp. 379-391.

[21] Parikinson and Spilker, Eds. Global Positioning System: Theory and Applications 1 . Published by AIAA

[22] J. P. Hespanha and A. S. Morse, "Stability of Switched Systems with Average Dwell-time," in Proc. of the $38^{\text {th }}$ Conference on Decision and Control, December 1999, pp. 2655-2660.

[23] D. Uberzon, J. P. Hespanha, and A. S. Morse, "Stability of Switched Linear Systems: a Lie-Algebraic Condition," Systems and Control Letters, Vol. 37, June 1999, pp. 117-122.

[24] G. Zhai, B. Hu, K. Yasuda, and A. N. Michel, "Stability Analysis of Switched Systems Including Unstable Subsystems: an Average Dwell time Approach," in Proc. of 2000 American Control Conference, June 2000 , pp. 200-204.

[25] G. Zhai, B. Hu, K. Yasuda, and A. N. Michel, "Disturbance Attenuation Properties of Time-Controlled Switched Systems," submitted to the European Control Conference, 2001.

[26] A. Hassibi, S. P. Boyd, and J. P. How, "Control of Asynchronous Dynamical Systems with Rate Constrains on Events," in Proc. of the 38 Conference on Decision and Control, December 1999, pp. 1345-1351 